Revista de Biología Marina y Oceanografía

Vol. 52, N²: 187-199, agosto 2017

DOI 10.4067/S0718-19572017000200001

\title{
REVISIÓN \\ Dimensiones espacial y temporal de los procesos de selección de hábitats críticos por las tortugas marinas
}

\author{
Spatial and temporal dimensions of the critical habitat selection processes by sea turtles
}

Eduardo Cuevas ${ }^{1}$

${ }^{1}$ Centro de Investigación y de Estudios Avanzados, Instituto Politécnico Nacional, Unidad Mérida, Departamento de Recursos del Mar, Carretera Antigua a Progreso Km. 6, S/N, C. P. 97310, Mérida, México. ecuevas@ cinvestav.mx

\begin{abstract}
Marine turtles are large vertebrates with a complex life cycle along which they occupy several habitats in their different stages. This complexity imposes challenges for the study and knowledge of their populations, and for decades numerous studies have targeted to understand the factors that determine the selection and use of the resources they require at their different stages. The inclusion of the spatial and temporal scales in the conception of the research efforts is of the highest transcendence to obtain robust and pertinent information about the habitat selection processes. The objective of this revision was to analyze different scales and approaches used to evaluate the selection processes of nesting beaches, nesting sites and feeding habitats by sea turtles, considering some of the biological, ecological and evolutive implications linked to these processes. Based on the available literature, some of the reported results are analyzed in terms of their implications for conservation and management of their populations and the critical habitats they occupy. The study of the processes involved in the selection and use of the critical habitats that sea turtles occupy is nowadays an active, pertinent and of highest relevance; fact that reassert the strategic and fundamental importance of long time monitoring studies, as the only way to evaluate in a convincing way alterations of their populations and habitats at different spatial and temporal scales.
\end{abstract}

Key words: Resources use, habitat selection, spatial scale, temporal scale

Resumen.- Las tortugas marinas son especies de grandes vertebrados que tienen un ciclo de vida complejo en el que ocupan diversos hábitats durante sus distintos estadios de vida. Tal complejidad impone retos para el estudio y conocimiento de sus poblaciones, y por décadas numerosas investigaciones se han dedicado a entender los factores que determinan la selección y uso de los recursos que requieren en sus diferentes etapas. La concepción a una escala espacial y temporal de los esfuerzos de investigación es de suma trascendencia para obtener información robusta y pertinente sobre los procesos de selección de sus hábitats. El objetivo de esta revisión fue analizar diferentes escalas y aproximaciones abordadas para la evaluación de los procesos de selección de playas de anidación, sitios de anidación y hábitats de alimentación por las tortugas marinas, considerando algunas de las implicaciones biológicas, ecológicas y evolutivas asociadas a dichos procesos. A partir de resultados publicados se analizan algunos de los resultados reportados y las implicaciones que estos procesos tienen en temas de conservación y manejo de sus poblaciones y hábitats críticos. El estudio de los procesos de selección y uso de los hábitats críticos que ocupan las tortugas marinas es una línea actualmente vigente, pertinente y de alta relevancia, a través de la cual se reitera la importancia estratégica y fundamental de estudios de monitoreo a largo plazo, única manera de evaluar de forma contundente modificaciones en sus poblaciones y hábitats a escalas espaciales y temporales distintas.

Palabras clave: Uso de recurso, selección de hábitat, escala espacial, escala temporal

\section{INTRODUCCIÓN}

Las tortugas marinas son reptiles adaptados a hábitats marinos y con un complejo ciclo de vida, además de ser organismos longevos, de maduración tardía e iteróparos, lo que las hace particularmente vulnerables a impactos antropogénicos de diversa índole (Bowen \& Karl 1997).

Tal complejidad representa algunos retos de última frontera para su estudio en diferentes disciplinas de la ecología y biología de la conservación, ya que ocupan numerosos hábitats a lo largo de su vida, y los cuales pueden localizarse a centenas y hasta millares de kilómetros de distancia entre ellos, lo que da lugar a la operación de una diversidad particular de procesos y mecanismos a diversas escalas espaciales y temporales, desde el nivel de organización de genes, hasta el de ecosistemas, como podría considerarse a las cuencas oceánicas.

El objetivo de este documento fue analizar diferentes niveles de organización, escalas y aproximaciones abordadas para la evaluación de los procesos de selección de playas, hábitats de desarrollo y hábitats de alimentación por las tortugas marinas, considerando algunas de las implicaciones biológicas, ecológicas y evolutivas asociadas a dichos procesos. Se hace referencia 
de forma particular a estudios realizados en el Golfo de México y Mar Caribe, por enfoque de trabajo y experiencia del autor.

Los procesos de selección de hábitats críticos por las tortugas marinas han sido estudiados por décadas, y aun hoy en día continúan siendo una línea de investigación vigente en lo cual se basa la pertinencia de la presente revisión (Rees et al. 2016), y se sostiene la necesidad de contar con referencias técnicas que fomenten la generación de información sobre esta línea de investigación.

\section{La Escala}

El término escala es uno de los conceptos más ambiguos y estudiados por la ciencia. Este término es utilizado tanto para referirse a la magnitud de un estudio (resolución geográfica, límites de las observaciones realizadas de un fenómeno) como al grado de detalle de una aproximación (su nivel de resolución geográfica) (Goodchild \& Quattrochi 1997).

Dos de las acepciones más frecuentes del término escala en ecología es la de 'dimensiones espacial y temporal a las cuales se observa un fenómeno en el campo' (Rietkerk et al. 2002), y 'el nivel de organización del ambiente en el cual podemos distinguir condiciones bióticas y abióticas heterogéneas' (Cassini 2013). Las interacciones entre organismos, y de estos con su ambiente ocurren en un amplio rango de niveles de organización y escalas espaciales y temporales (Neuhauser 2001), aspecto sobre los cuales el desarrollo de la teoría jerárquica en ecología ha enseñado cómo los procesos y sus restricciones cambian en cada uno de ellos, por lo que la escala a la que observamos un fenómeno a un nivel específico es determinante para su entendimiento (Turner et al. 1999). En lo general se reconoce que el concepto de escala se relaciona principalmente con patrones, mientras que los niveles están más relacionados con procesos (Cassini 2013).

Uno de los conceptos principales de la teoría jerárquica señala que las escalas espacial y temporal tienden a variar de forma conjunta (co-variar), es decir, que procesos que operan en niveles altos y escalas temporales largas, también operan sobre escalas espaciales grandes, debido en parte a que el espacio y el tiempo están ligados por mecanismos ecológicos operantes (Goodchild \& Quattrochi 1997).

El concepto de escala se compone de 3 elementos esenciales: tamaño de grano (el tamaño de la unidad de muestreo utilizada), extensión (el área total o volumen cubierto por el área de estudio) y el intervalo de muestreo (la distancia promedio entre las unidades muestrales realizadas). El grano y la extensión pueden ser características intrínsecas del fenómeno observado, o también características extrínsecas de la observación realizada de ellos (Pereira 2002). Para fines de esta contribución se analizan los procesos desde una aproximación primordialmente jerárquica, como se ha reconocido por diversos autores, en la cual se considera que el grano de una escala es menor que el grano de otra, y la extensión de la primera también es menor que la de la segunda.

Se ha sugerido que no existe una escala 'apropiada' para el estudio y descripción de un fenómeno dado; y que sin importar la escala definida para las observaciones es necesario demostrar que el fenómeno estudiado opera realmente a la escala seleccionada (Pech et al. 2007). Se ha generalizado el hecho de reconocer que cada fenómeno tiene una escala distintiva y característica que puede ser reconocida a priori, aunque al establecer de esta manera la escala de observación del fenómeno en estudio se corre el riesgo de no considerar aspectos que operen a escalas diferentes y resulte en una descripción inadecuada (Rietkerk et al. 2002).

En aquellos casos en los que no es posible establecer de forma contundente esta escala inicial para el estudio de fenómenos específicos se sugiere abordarlos con una aproximación de escalas múltiples para incluir y cuantificar la mayor variabilidad posible del fenómeno estudiado (Rietkerk et al. 2002). Se remarca la importancia de las escalas en cualquier estudio ecológico que se realice, por lo que deben ser tomadas en consideración en el diseño, ejecución e interpretación de las investigaciones.

\section{SELECCIÓN Y USO DE LOS RECURSOS POR LOS ANIMALES, CONCEPTOS Y SUPUESTOS}

La identificación y evaluación de la disponibilidad de los recursos utilizados por las poblaciones, así como el entendimiento de cómo éstas responden a su variabilidad espacial y temporal a diferentes escalas reviste una gran importancia para el diseño de investigaciones ecológicas y programas de monitoreo, en especial para poblaciones de especies vulnerables y en peligro de extinción como es el caso de las tortugas marinas (Boyce \& McDonald 1999, IUCN 2017).

La evaluación del uso de recursos por depredadores ubicados en la parte alta de las redes tróficas es de especial importancia dado que estos depredadores influyen en la distribución y abundancia de presas y otras especies de forma directa o indirecta (Heithaus et al. 2006). Este es el caso de las tortugas marinas, las cuales se sitúan en niveles altos de las redes tróficas de las que forman parte, y es un punto clave de su relevancia (Gulko \& Eckert 2004).

Uno de los principales supuestos en la evaluación del uso de recursos por los animales es que las especies elegirán los recursos que mejor satisfagan sus necesidades básicas, y que los recursos de mejor calidad serán preferidos sobre aquellos 
de menor calidad, asociándose los hábitats de alta calidad con un mayor éxito reproductivo en comparación con hábitats de menor calidad (Julliard 2000).

Por ejemplo, en los modelos básicos de selección de hábitat se asume que, para cada decisión de selección un animal evalúa todos los hábitats disponibles y escoge el hábitat con la más alta calidad. En estos modelos, si una localidad de mayor calidad queda disponible, un animal con comportamiento óptimo cambiará a ese nuevo hábitat; sin embargo, esta premisa no siempre se puede generalizar. Aquellos animales que no se muevan al nuevo hábitat demostrarían lo que se conoce como fidelidad al sitio (Switzer 1993), y son incluso más susceptibles de quedar en una trampa ecológica con condiciones subóptimas para sus requerimientos (Gilroy \& Sutherland 2007). Frecuentemente se reportan casos en los que las tortugas marinas caen en trampas ecológicas debido a características evolutivas como es la fidelidad a sus hábitats críticos, por lo que este argumento cobra mayor relevancia para estas especies.

Manly et al. (2002) definen el uso de un recurso (espacio, alimento, sustrato, etc.) como la cantidad de recurso que es utilizado por un animal (o población de animales) en un período de tiempo establecido; y la disponibilidad de un recurso como la cantidad de éste que se encuentra accesible para el animal durante el período de tiempo establecido. Por su parte, Beyer et al. (2010) definen el uso de hábitat (recurso) como la proporción del tiempo que los animales pasan en un hábitat particular. En ambas definiciones es evidente el uso de una métrica para la medición del uso, para lo que frecuentemente se utiliza el tiempo.

Relacionados con estos conceptos, en ocasiones los términos selección y preferencia son utilizados de manera indistinta, cuando en realidad son diferentes. La selección es el proceso en el cual un animal escoge un recurso, y la preferencia es la probabilidad de que un recurso sea seleccionado sobre otros disponibles en iguales condiciones de accesibilidad (Manley et al. 2002), o la probabilidad de que un animal elija un recurso dado cuando se le ofrecen alternativas en iguales condiciones (Beyer et al. 2010).

La selección de un recurso se presenta de manera jerárquica, iniciando desde el rango geográfico de distribución de una especie, el ámbito hogareño de un individuo dentro de un rango geográfico, al uso de condiciones generales (hábitat) dentro del ámbito hogareño, y a la selección de elementos particulares (organismos presa) dentro de los hábitats (Manley et al. 2002). Para fines de esta revisión, se considera Hábitat a la región en el espacio ambiental que se compone de múltiples dimensiones, cada una representando variables bióticas o abióticas (Beyer et al. 2010). Normalmente la extensión del hábitat utilizado por los animales aporta una aproximación a la calidad y abundancia del recurso utilizado en esas áreas, lo cual refleja la salud del hábitat, aun cuando existen excepciones a esta afirmación (Boyce \& McDonald 1999).

\section{Procesos de Selección por los animales}

Entre los factores reportados con influencia importante en el proceso de selección de recursos se encuentran: la densidad poblacional, competencia inter-específica, la selección natural, la composición química o textura del alimento, la heredabilidad, la depredación y la estructura paisajística.

Se ha propuesto que en la evaluación de fenómenos ecológicos se deben considerar al menos 3 niveles de escala al mismo tiempo: la que se quiere evaluar; una abajo, la cual aporta información sobre los mecanismos que actúan en ese fenómeno o proceso; y una arriba, la cual brinda información acerca del contexto en el que se da el fenómeno de estudio.

Es evidente que no se puede dar un proceso de selección de recursos en un ambiente totalmente homogéneo, pero no lo es el cómo la heterogeneidad espacial y/o temporal de los recursos puede influir directamente en el proceso de selección por el animal (Boyce \& McDonald 1999).

\section{Selección de hábitats CRÍticos POR laS TORTUgaS MARINAS}

Diversos autores han propuesto que el proceso de selección del sitio de anidación se presenta en una jerarquía de escalas ordinales de 3 niveles: una gruesa, una media y una fina; con las cuales también interactúa un cuarto componente que es la escala temporal (Burskirk \& Millspaugh 2006), una de las principales fuentes de variabilidad en la disponibilidad y calidad de los hábitats (Kamel \& Mrosovsky 2006).

La vasta mayoría de los estudios realizados sobre selección de hábitats se centran en la playa de anidación (más del 99\%), dejando a un lado la generación de información básica sobre los procesos, patrones y biología de las tortugas marinas en hábitats críticos acuáticos (Byles 1991). En este vacío de información se incluye el problema de un escaso conocimiento sobre el proceso de selección del hábitat de alimentación.

Al respecto de la marcada diferencia en la cantidad de información y conocimiento existente entre los dos procesos de selección de hábitat, de manera parcial se debería a que los estudios de vertebrados mayores, con tiempos de vida largos, rangos geográficos de distribución muy amplios y capacidades de migrar miles de kilómetros entre hábitats utilizados, son altamente costosos, requiriendo personal y equipamiento especializado para su realización (Byles 1991). Esta escasez 
de estudios en ambientes marinos y oceánicos se refleja en la cantidad de literatura disponible sobre los procesos de selección de los hábitats críticos abordados.

\section{SELECCión dE HÁbITAT dE ANIDACIÓN}

Los procesos de selección de recursos por las tortugas marinas, así como los patrones ecológicos en los que se ven inmersas, están influidos por la escala a la cual se aborde el fenómeno. En este proceso de selección de hábitats críticos, existen diferentes mecanismos y variables que influyen de manera directa e indirecta en la selección del recurso por las tortugas, y sobre los cuales Kelly et al. (2017) realizaron una revisión minuciosa de publicaciones al respecto.

La variación individual del comportamiento de selección de hábitat es relevante para muchos procesos a gran escala como son los evolutivos y ecológicos, ya que sus preferencias de hábitat pueden desempeñar un papel importante en la dinámica de metapoblaciones, adaptación local y especiación simpátrica (Refsnider \& Janzen 2010).

\section{EsCALA ESPACIAL GRUESA}

Por escala gruesa se hace referencia a la primera escala de aproximación, y la más general, en el estudio y evaluación del proceso de selección de playa de anidación por las tortugas marinas, el cual ha sido referido a mecanismos operantes a grandes escalas espaciales (a nivel de continentes, océanos e incluso de todo el planeta) y temporales (a nivel de selección natural, evolución y especiación), ejemplos de éstos son la filopatría y los mecanismos de navegación, incluyendo aquellos utilizados por las tortugas para la migración y su establecimiento en los sitios de alimentación (Stiebens et al. 2013).

\section{Filopatría}

La filopatría es el fenómeno por el cual los individuos de especies particulares regresan a reproducirse a sus sitios de nacimiento, en este caso emergencia, y ha sido ampliamente documentado para diferentes grupos taxonómicos (Feldheim et al. 2014).

La filopatría induce una distinción genética entre poblaciones por su fidelidad a una región, y en la cual también sus padres se reprodujeron, minimizando así el intercambio genético entre poblaciones y evitando una homogenización genética entre poblaciones distintas, a la vez que provee de una considerable autonomía demográfica para las poblaciones en lo que respecta a la reproducción (Avise 1998).

El estudio de la filopatría se ha realizado principalmente utilizando marcadores moleculares, como son los microsatélites y el ADN mitocondrial, para cuantificar la tasa de retorno de hembras anidantes a sus playas de emergencia (Bass 1996, Lee et al. 2007).

Con este tipo de estudios se ha demostrado que tanto las hembras como los machos migran de regreso a sus regiones de eclosión para reproducirse, y en el caso de las hembras, a anidar. Estas migraciones se dan a una escala geográfica amplia, variando las distancias recorridas dependiendo de la especie, pero pudiendo migrar desde unas pocas decenas de kilómetros hasta miles de kilómetros para llegar a sus sitios de reproducción (Godley et al. 2007).

De manera general, este tipo de aproximación opera bajo el supuesto de que una baja diversidad de haplotipos es reflejo de la fidelidad al sitio de anidación, encontrándose que hembras anidantes en la misma localidad tienen una mayor similitud genética entre ellas que con hembras de otras localidades. A escala amplia espacial y temporal, estos estudios son efectivos; sin embargo, en el caso de las tortugas marinas, a una escala fina no se ha tenido éxito en estudiar y determinar de forma contundente este mecanismo particular (Stiebens et al. 2013).

Se ha argumentado que la filopatría es una desventaja evolutiva para las tortugas marinas, siendo la mayor implicación la extinción local de poblaciones anidantes, representando una pérdida irreparable de variación genética de la especie (Lee et al. 2007); sin embargo, esta hipótesis ha sido discutida por otros estudios en los que se demuestra que en realidad contribuye a mantener la diversidad genética dentro y entre poblaciones (Stiebens et al. 2013). Algunas de las implicaciones evolutivas de la filopatría se reflejan en el proceso de selección del sitio de anidación y en la proporción de sexos de las crías, dado que las tortugas marinas tienen una determinación de sexo por temperatura (DST) (Refsnider \& Janzen 2010).

La filopatría también tiene ventajas sobre el éxito reproductivo de las especies que presentan esta característica, proveyendo de una solución a situaciones de alto riesgo, como es la selección de hábitats críticos para reproducción al asegurar que aun cuando los organismos se dispersen a través de muy largas distancia, estos puedan reconocer de forma rápida y confiable al menos un tipo de hábitat adecuado para su anidación entre muchos otros, probablemente de menor calidad, que convergen en un mismo paisaje heterogéneo (Davis \& Stamps 2004). De igual forma, ayuda a mantener su potencial adaptativo e influye en las proporciones sexuales de las poblaciones (Nishizawa et al. 2011, Stiebens et al. 2013).

En las especies que presentan filopatría, como las tortugas marinas, las hijas utilizan los hábitats de anidación utilizados por sus madres, esto implica que las hijas tienen una preferencia ya sea por una localidad geográfica en particular (escala espacial gruesa) o por un tipo de microhábitat similar al seleccionado 
por sus madres (escala espacial fina), y el cual ha sido probado de forma exitosa en el pasado por la misma madre (Valenzuela \& Janzen 2001).

Para el Golfo de México no se encontraron estudios a escala gruesa con el objetivo específico de evaluar la filopatría de las tortugas adultas reproductoras; sin embargo, sí existen estudios de genética poblacional que de forma indirecta aportan información sobre la composición y estructura poblacional dentro de poblaciones anidantes, al igual que enseñan un panorama más completo de los haplotipos y su diversidad presente en la región, particularmente para el sureste de México (Barceló-Celis 2015, González-Garza et al. 2015).

\section{MeCanismo de NAVEGaCión}

Las tortugas marinas son capaces de realizar largas travesías como migraciones entre hábitats críticos, teniendo la capacidad de hacer incluso viajes transoceánicos entre sus áreas de alimentación y desarrollo hasta llegar a sus playas de anidación (Bowen \& Karl 1997).

La capacidad de navegación con exactitud que presentan animales como las tortugas marinas, langostas espinosas, tiburones y aves migratorias ha sido tema de diversas investigaciones multidisciplinarias que han brindado evidencia clave sobre algunos de los mecanismos que utilizan. En el caso particular de las tortugas marinas, las explicaciones para estas capacidades de navegación de los individuos adultos se basan en información generada particularmente para individuos juveniles y crías (Brothers \& Lohman 2015).

El principal mecanismo elucidado para explicar la navegación de las tortugas marinas es la percepción del campo magnético de la Tierra, con lo que las tortugas pueden estimar su posición en la tierra y navegar a sus áreas de alimentación y anidación. Se ha demostrado que las tortugas crías poseen un sentido de compás magnético que les permite establecer y mantener rumbos relativos al campo magnético de la Tierra (Diego-Rasilla 2004, Luschi et al. 2007).

Las tortugas tienen la capacidad de percibir, además del ángulo de inclinación de la tierra, al menos otros 3 parámetros magnéticos: (1) la intensidad (fuerza) del campo total, (2) la intensidad del campo horizontal, (3) la intensidad del campo vertical (Lohman et al. 2007). En principio, la habilidad para percibir estas características del campo magnético podría permitir a las tortugas identificar áreas particulares de playas continentales a una escala global (Lohman et al. 2003).

De gran relevancia es el hecho reportado por Lohman et al. (2012) en donde aseguran que las tortugas no necesitan de una experiencia migratoria para reconocer el camino que deben recorrer en su migración, y que incluso las crías no requieren pasar por una secuencia de campos magnéticos previo a responder a estímulos magnéticos con los que nunca antes habían estado en contacto. Esta información sobre los mecanismos de navegación de las tortugas es de alta relevancia para el entendimiento de los procesos de selección de sus hábitats de anidación, desarrollo y alimentación, siendo ampliamente aceptado que es en realidad una combinación de mecanismos como la filopatría, magnetismo de la Tierra, estímulos químicos e incluso visuales, los que auxilian y dirigen a las tortugas durante sus recorridos de largas distancias.

\section{ESCALA ESPACIAL MEDIA}

La escala media en el proceso de selección del hábitat de anidación de las tortugas marinas hace referencia a una extensión espacial que se restringe a un área de decenas de kilómetros, en zonas aledañas a las playas de anidación, así como características de la misma playa (Kelly et al. 2017).

Esta escala es una de las más estudiadas y enigmáticas para los especialistas en tortugas marinas de todo el mundo, ya que incluye el período entre la llegada de la hembra a los alrededores de la playa de anidación y su salida a la franja arenosa para depositar sus huevos, segmentos del ciclo de vida de las tortugas marinas en los que se concentra más del $90 \%$ de los esfuerzos de investigación y conservación alrededor del mundo.

Un mejor entendimiento de la biología de anidación de las tortugas marinas se vincula con la habilidad de evaluar los efectos de diferentes perturbaciones antropogénicas sobre el éxito reproductivo de las tortugas a diferentes escalas espaciales y temporales, mejorando así la eficiencia de nuestros planes de conservación (Mazaris et al. 2006). En particular, la selección del sitio de anidación para las tortugas marinas reviste una gran importancia debido a la ausencia de cuidado parental, ya que la implicación de seleccionar un sitio de anidación de baja calidad no puede ser compensada por el comportamiento de cuidado de los padres (Refsnider \& Janzen 2010).

El éxito de eclosión y emergencia de las crías está asociado a la zona de la playa de anidación en la que la hembra deposita sus huevos, así como las condiciones prevalecientes en el microhábitat dentro del nido, influyendo en el nivel de riesgo de inundación de los nidos, la facilidad de acceso de las crías hacia el mar, así como el riesgo de depredación de crías y huevos (Mazaris et al. 2006). Con estas consideraciones, la aproximación para la evaluación de selección del hábitat de anidación a escala media tiene implicaciones mayores en la viabilidad y éxito reproductivo de las poblaciones de las tortugas marinas. 
Diversos estudios han identificado múltiples parámetros físicos y químicos asociados con la selección del sitio de anidación a una escala media de playa de anidación (entre 20 y $50 \mathrm{~km}$ de extensión en promedio): ancho de la playa, pendiente y cobertura vegetal de la playa, cantidad de luz en playa, régimen de mareas, entre otras (Ficetola 2007, Pfaller et al. 2008). Para mayor detalle sobre esta etapa específica, Kelly et al. (2017) entregan amplia información de este listado de variables.

Otros de los factores asociados a este proceso de selección es la dinámica de las playas a lo largo del tiempo, régimen de corrientes oceánicas, temperatura oceánica, del aire y arena en las playas de anidación, entre otros (Tiwari et al. 2005). También se ha mostrado que el clima en algunas ocasiones tiene influencia a esta escala, tanto en la ocurrencia de la temporada de reproducción como en el inicio, duración y fin a la anidación de las tortugas (Mazaris et al. 2013, Neeman et al. 2015, ÁlvarezAcosta 2016).

Sin embargo, a pesar de la enorme cantidad de trabajos realizados en este tema, la comprensión sobre los procesos detallados que controlan la selección del sitio de anidación a una escala espacial media y fina es aún limitada. Lo que sí está claro es que las tortugas marinas utilizan diferentes grupos de variables ambientales en diferentes momentos del proceso de selección del sitio de anidación en sus distintas escalas espaciales (Mazaris et al. 2006).

Provancha \& Ehrhart (1987) en Florida, Estados Unidos, y Mazaris et al. (2006) en Grecia, evaluaron la influencia de la morfología de la playa de anidación sobre la actividad de las hembras de tortuga caguama (Caretta caretta), y reportan preferencias por segmentos de playa con pendientes suaves y angostas, confirmando la importancia de las variables pendiente de playa y ancho de la misma.

Por su parte, Horrocks \& Scott (1991) realizaron una evaluación de la influencia de la ubicación del nido en el perfil de playa sobre el éxito de la nidada de tortugas carey (Eretmochelys imbricata) en Barbados, en la cual encontraron que las tortugas carey prefieren playas con bajo nivel de oleaje y pendientes pronunciadas. Por el contrario, Ficetola (2007) reportó que las hembras carey prefieren en playas con pendientes suaves, con poca presencia de rocas en la playa y, particularmente, una alta cobertura vegetal en la misma. Cuevas et al. (2010) reportan una preferencia de condiciones de playa de anidación en Yucatán, México distintas entre las especies carey (E. imbricata) y blanca (C. mydas), con las tortugas blancas prefiriendo sitios con pendiente pronunciada y angostas, y las tortugas carey seleccionando sitios con pendiente de playa suave $\mathrm{y}$ anchas.
Una de las razones por las que este conocimiento es de alta relevancia es por la medición de impactos causados por alteraciones en sus hábitats. Rumbold et al. (2001) cuantificaron y evaluaron el efecto del relleno de playas sobre la actividad de anidación de tortugas caguama ( $C$. caretta) en el estado de Florida en Estados Unidos, tomando en consideración también la dimensión temporal. Evaluaron dos temporadas de anidación consecutivas después de la alteración de la playa, reportaron que la actividad de anidación de tortugas hembras fue significativamente menor en los segmentos de playa rellenados, debido principalmente a modificaciones de características de escala media como son ancho de la playa y morfología de la misma.

La contraposición de algunas preferencias reportadas para una misma especie, es un ejemplo de las complicaciones que pueden surgir por la variabilidad poblacional e individual en el proceso de selección y preferencia de playas de anidación.

\section{ESCALA ESPACIAL FINA}

Los mecanismos inmersos en este nivel de escala espacial se encuentran en la última porción de la secuencia del proceso de selección de hábitat de anidación, y la pertinencia de estos estudios básicos es vigente, requiriéndose evaluaciones sobre aspectos básicos de la biología de estas especies. Este nivel espacial del proceso de selección incluye ya el período de tiempo una vez que la hembra se encuentra fuera del agua y está reconociendo y rastreando la playa de anidación en busca del sitio específico para depositar sus huevos.

La selección del sitio específico de anidación se considera como un proceso adaptativo de compensación entre el costo de búsqueda del sitio de anidación (tanto en términos de gasto energético como de riesgo de depredación) y los beneficios reproductivos de seleccionar un sitio adecuado para la incubación exitosa del nido (Wood \& Bjorndal 2000).

Aquí, comportamientos de rastreos específicos, así como sensores y percepciones detalladas del medio ambiente son factores determinantes para la selección del sitio de anidación. A esta escala operan, principalmente variables de microhábitat como son tamaño de grano, clasificación del sedimento, $\mathrm{pH}$, conductividad, temperatura de la superficie de la arena, contenido de materia orgánica, ángulo de inclinación, humedad, compactación del sedimento (Wood \& Bjorndal 2000). Estas variables afectan de diferente forma la supervivencia de los embriones, el tamaño de las crías, su crecimiento, comportamiento y determinación de sexo.

Mortimer(1990) evaluó la influencia de características físicas y químicas de la arena en playas sobre el comportamiento de 
anidación y éxito de los nidos depositados de tortuga blanca (C. mydas) en Isla Ascensión, para la cual reportó una relación positiva entre el diámetro promedio de la partícula de arena con la mortalidad de embriones, y el éxito del nido está correlacionado de forma positiva con la profundidad de la cámara, y negativa con la conductividad y porosidad de la arena.

En el mismo estudio antes citado, Horrocks \& Scott (1991) encontraron para tortugas carey (E. imbricata) en Barbados, que al aumentar el nivel de compactación del sedimento el éxito de emergencia de los nidos disminuye significativamente; mientras que, en Florida, Estados Unidos Wood \& Bjorndal (2000) demostraron que la humedad y salinidad de la superficie de la playa son potenciales pistas utilizadas por las hembras para la selección del sitio de anidación por las tortugas caguama (C. caretta).

Estudios ecológicos básicos realizados a esta escala espacial son de gran relevancia para el establecimiento de líneas base de las condiciones físicas y químicas de los sedimentos en playas de anidación, proveyendo así de herramientas técnicas, científicas y robustas para la definición de criterios a utilizar en política pública por autoridades.

\section{Selección de hábitats de alimentación y DESARROLLO POR TORTUGAS MARINAS}

Al igual que la selección del hábitat de anidación por las tortugas hembras anidantes, el proceso de selección y establecimiento de las tortugas marinas en sus hábitats de alimentación implica factores intrínsecos y extrínsecos a los individuos o poblaciones estudiadas. El estudio de los estadios y hábitats críticos meramente acuáticos de las tortugas marinas han sido pobremente estudiados, debido principalmente a los altos costos que implican el equipo y personal especializado y capacitado para estas labores.

Para propósitos del presente documento, también se clasificaron los estudios referidos en 3 escalas de magnitud espacial, incluyendo un componente temporal transversal a todas ellas, y se resumen algunas de las implicaciones y ejemplos de contribuciones para cada una de las 3 aproximaciones mencionadas.

\section{ESCALA ESPACIAL GRUESA}

Este enfoque opera a escala de grandes regiones, como son cuencas oceánicas e incluso escalas continentales, para las que se recurre de disciplinas y ciencias como la genética, oceanografía y geofísica, con el fin de generar información que permita conocer con la mayor precisión posible los procesos que actúan a esta escala en la selección y establecimiento de individuos juveniles y adultos de tortugas marinas en sus hábitats de alimentación y desarrollo alrededor del mundo.

En la literatura disponible, se presentan varios ejemplos de aproximaciones que incluyen de manera integrada datos de genética poblacional, oceanografía y características bióticas para la definición del origen de concentraciones de tortugas marinas específicas en áreas de alimentación reconocidas (Blumenthal et al. 2009, Putman et al. 2012, 2014).

La mayoría de estos estudios buscan definir qué playas de anidación contribuyen con organismos y cuáles áreas son de alimentación; información que permitiría responder a problemas de escala espacial gruesa y media como es el origen poblacional de los organismos capturados de forma incidental en pesquerías (Bowen et al. 2007).

Bass et al. (2007) evaluaron los cambios en la composición poblacional de una agregación de tortugas caguama (C. caretta) en su área de alimentación, considerando la variabilidad a lo largo del tiempo. Este estudio es un ejemplo del amplio reconocimiento de la necesidad de monitoreo a largo plazo en hábitats críticos acuáticos, con el objetivo principal de rastrear el estado de conservación y condición de las tortugas; así como evaluar tendencias poblacionales y generar información sobre la historia de vida de las especies.

Hawkes et al. (2006) evaluaron las estrategias de forrajeo de tortugas caguama (C. caretta) utilizando telemetría satelital en Cabo Verde, África del Oeste, para las cuales definieron 2 estrategias diferentes de forrajeo, unas alimentándose en aguas costeras y las otras en zonas oceánicas, con lo cual se resalta la variabilidad por segmentos poblacionales e individual en la escala espacial del uso de las zonas de alimentación, registrándose organismos cuyo ámbito geográfico no rebasa las decenas de kilómetros, mientras otros cubren cientos y hasta miles de kilómetros para establecerse en sus áreas de alimentación (Godley et al. 2007).

Por otra parte, Engstrom et al. (2002) y Bowen et al. (2007) realizaron evaluaciones del origen de los organismos encontrados en áreas de alimentación de tortugas marinas en el Golfo de México y Mar Caribe para las especies de tortuga carey (E. imbricata) y caguama (C. caretta). Entre las aportaciones de estas investigaciones tenemos la afirmación de que la confluencia de haplotipos de diferentes playas de anidación en una misma área de alimentación podría ser una característica general de la biología poblacional de las tortugas marinas, mostrando las implicaciones a escala espacial de la conservación de estas especies.

Putman et al. (2012) resaltan en sus conclusiones el hallazgo de que las tortugas crías y juveniles tempranas pueden 
determinar el rumbo de su migración, contraponiéndose al supuesto de que sus movimientos estaban dictados únicamente por las corrientes marinas superficiales, con lo que se demuestra su capacidad para procurar alcanzar hábitats con condiciones apropiadas para su desarrollo.

De igual forma sugieren que el asentamiento de los individuos juveniles a ambientes bentónicos no se presenta de una manera aleatoria, y que dicha selectividad puede responder a factores de heredabilidad y filopatría de los individuos. Esta preferencia por sitios cercanos a su playa de anidación demostrada para algunas especies (Bowen et al. 2007) implica alguno de los mecanismos de navegación mencionados en secciones anteriores, como es la navegación por campos magnéticos como es el caso de la especie E. imbricata (Avens \& Lohman 2004).

De manera general, una de las aportaciones de mayor trascendencia, y que comparten con otros autores (Bass 1996, Norrgard \& Graves 1996, Lahanas et al. 1998), es la hipótesis de que las grandes colonias de tortugas anidantes contribuyen en mayor proporción con individuos establecidos en áreas de alimentación que las poblaciones pequeñas; y que colonias anidantes cercanas al área de alimentación contribuyen en mayor proporción que las más lejanas.

\section{ESCALA ESPACIAL MEDIA}

A esta escala espacial se hace referencia a comportamientos de buceo en zonas de alimentación, caracterizaciones espaciales y ecológicas de hábitats de alimentación, definición de ámbitos hogareños, uso y selección de parches de hábitats y evaluaciones de la distribución y abundancia de organismos en áreas de estudio con extensiones de decenas de kilómetros.

Una de las aproximaciones más generales es la definición del ámbito hogareño, esto es, la delimitación de las zonas de alimentación de las tortugas marinas una vez establecidas en el destino final de su migración. Este tipo de estudios son mayormente realizados utilizando técnicas de telemetría, ya sea satelital o por radio. Hoy en día son numerosas las investigaciones que utilizan esta tecnología, sosteniendo un incremento notable en la última década (Jeffers \& Godley 2016), por lo que en esta contribución solamente se hace mención de algunos estudios particulares.

Uno de los primeros estudios publicados, evaluando el ámbito hogareño de tortugas marinas en sus áreas de alimentación, es el de Renaud \& Carpenter (1994) para tortugas caguama ( $C$. caretta) en el norte del Golfo de México. Se ha reportado que esta especie de tortuga marina utiliza grandes extensiones de hábitats de alimentación, probablemente debido a que la distribución espacial de los organismos que conforman la base de su dieta promueva ese comportamiento.
En años posteriores, Seminoff et al. (2002) evaluaron el ámbito hogareño de tortugas blancas ( $C$. mydas) juveniles en áreas de alimentación en el Mar de Cortés, donde encontraron diferencias en el uso del hábitat por tallas de los individuos entre el día y la noche, demostrando la alta relevancia de este tipo de estudios para la delimitación de zonas específicas y la implementación de acciones estratégicas de conservación de la especie y su hábitat.

En el sur del Golfo de México, Cuevas et al. (2008) y Méndez et al. (2013) cuantificaron el ámbito hogareño para tortugas de carey (E. imbricata) y tortuga verde post-anidantes en la península de Yucatán, México. Identificaron áreas de alta relevancia para la alimentación y estancia de tortugas de carey en la región, reportando diferencias en la extensión de estas áreas de alimentación.

Shaver et al. (2016) identificaron los principales patrones migratorios de las tortugas lora (Lepidochelys kempii) en el Golfo de México, mostrando los caminos que recorren entre sus playas de anidación y sus zonas de alimentación, tanto en el norte como en el sur de este ecosistema.

Brill et al. (1995) presentan una de las primeras publicaciones sobre el comportamiento diferencial de tortugas blanca (Chelonia mydas) en hábitats de alimentación en las islas de Hawái, Estados Unidos, en donde determinaron los movimientos diurnos, uso de hábitat e intervalos de buceo de tortugas blancas juveniles. Mientras que van Dam \& Diez (1996) registraron también usos diferenciales del hábitat de alimentación de tortugas de carey juveniles en Puerto Rico, empleando una aproximación de escala espacial media.

Cuevas et al. (2007) realizaron una evaluación del uso de hábitat como área de alimentación de la tortuga carey en Yucatán, México, en el cual reportaron diferencias en el uso del hábitat entre día y la noche, así como la ocupación de diferentes tipos de hábitat submarino.

En este nivel de escala espacial también se incluyen estudios relacionados con tasa de crecimiento dentro de hábitats de alimentación específicos, factor biológico clave para la evaluación del nivel de éxito de la selección del hábitat de anidación al estar relacionado de forma directa con la calidad del hábitat seleccionado para establecerse (Bjorndal et al. 2016).

\section{ESCALA ESPACIAL FINA}

Igual que su escala homóloga en el proceso de selección de hábitat de anidación, esta escala es la de menor extensión y en la que operan mecanismos cuya extensión incluso puede ser el mismo individuo de estudio. En esta escala se incluyen 
evaluaciones específicas de la dieta de tortugas marinas y el nivel trófico en el que se encuentran dentro de la red trófica operante en los sistemas que ocupan. Este tipo de aproximaciones requiere de conocimientos y experiencias altamente especializados, dado que usualmente el muestreo se da en el mismo individuo, por lo que se requiere de experiencia en dicha toma de datos para evitar cualquier tipo de impacto negativo sobre los individuos o poblaciones muestreadas.

León \& Bjorndal (2002) evaluaron la función ecológica de las tortugas carey (E. imbricata) en los ecosistemas marinos, dada su especificidad de dieta a base de esponjas, como predadores importantes en sistemas arrecifales. Goatley et al. (2012) reafirmaron un efecto positivo indirecto sobre los corales al alimentarse de uno de sus principales grupos competidores, al mismo tiempo que se incrementa la biodiversidad bentónica global del arrecife.

Un ejemplo más de una aproximación a escala espacial fina sobre la selección del hábitat de alimentación de las tortugas marinas, es la evaluación del nivel trófico de las tortugas marinas en las redes tróficas. Para esto se utilizan métodos que cuantifican isótopos estables, principalmente de carbono y nitrógeno, generándose también información acerca del tipo de alimento que está siendo consumido por esas poblaciones (Ceriani et al. 2014, Vander-Zanden et al. 2014).

Este tipo de análisis es altamente especializado por lo que no está ampliamente distribuida la aplicación de esta metodología para las tortugas marinas, aun cuando es una herramienta de invaluable utilidad para el estudio y entendimiento de patrones espaciales y temporales a diferentes escalas de operación.

\section{COMPONENTE ESCALA TEMPORAL}

En ambos procesos de selección de hábitat de anidación y de alimentación se mencionó la existencia de un componente transversal a las 3 escalas espaciales comentadas, la escala temporal. Ésta es abordada de manera poco detallada en algunos de los estudios referidos en los procesos de selección dentro del presente documento.

La vasta mayoría de las aportaciones citadas en este documento hacen referencia a un solo momento, una temporada de anidación, una imagen instantánea del proceso. Sin embargo, los procesos de selección de los hábitats se presentan dentro de un contexto de variabilidad temporal en todas las escalas espaciales. Y es que este componente temporal tiene una influencia sobre todas y cada una de las escalas espaciales que se quieran abordar en el estudio de la ecología de las tortugas marinas y sus hábitats críticos; la cual incluye factores de alta relevancia como son tormentas y huracanes, a escala media; cambio climático y tiempos geológicos.
Una de las características que ha sido evaluada con una aproximación de escala temporal es la repetición de preferencia de sitio de anidación de una hembra entre diferentes años de anidación (Kamel \& Mrosovsky 2006). Esta aproximación con tortugas carey (E. imbricata) anidadoras sirvió para evaluar tanto la repetitividad de la selección del sitio, como el nivel de heredabilidad de las preferencias de sitio de anidación entre generaciones.

Un conjunto de preguntas de investigación pendientes tales como si los procesos, variables, condiciones, composiciones, estructura y equilibrio cambian a lo largo del tiempo (en corto, mediano o largo plazo). Alteraciones de alto impacto, como es el cambio climático, traen consecuencias tanto ecológicas inmediatas y de mediano plazo, como evolutivas y de escalas temporales grandes (Schlaepfer et al. 2002, Hawkes et al. 2007).

Es por estas razones que la escala temporal debe ser considerada en todas las evaluaciones de selección de hábitat crítico por las tortugas marinas, sin importar la escala o aproximación utilizada en el estudio, dado que las tortugas y sus hábitats están envueltos en una dinámica de variabilidad, cuyo ritmo muchas veces es dirigido por el tiempo.

\section{CONCLUSIONES}

El estudio de los mecanismos de selección de hábitats críticos por las tortugas marinas es una línea de investigación vigente y la cual cuenta con herramientas tecnológicas de vanguardia para la colecta de datos en campo y en laboratorio. La información que se genera es de relevancia estratégica para la conservación y manejo de sus poblaciones y hábitats críticos, información que debe ser transferida de manera óptima en los responsables de toma de decisiones para su uso en el diseño de políticas públicas, incluyendo la definición de estrategias voluntarias y oficiales de protección de áreas claves de agregación y migración de estas especies y que involucre también la protección de las poblaciones vulnerables y especies en peligro de extinción de tortugas marinas.

Es clave considerar una aproximación de escalas espacial y temporal múltiple para una mejor evaluación y entendimiento de los mecanismos de interacción que ocurren entre individuos y especies de tortugas marinas, así como con los hábitats que ocupan, incluyendo tanto los procesos físicos como los biológicos dentro de los ecosistemas. Esto lleva a reconocer la importancia estratégica y fundamental de estudios de monitoreo a largo plazo, principal manera de evaluar en forma contundente modificaciones en sus poblaciones y hábitats. 


\section{Agradecimientos}

Al Consejo Nacional de Ciencia y Tecnología (CONACYT) de México por la beca de Doctorado en Ciencias Marinas otorgada al autor de esta contribución (\#44779) para su doctorado en ciencias marinas. Al Centro de Investigación y de Estudios Avanzados del Instituto Politécnico Nacional (CINVESTAV), Unidad Mérida, por las facilidades para participar en su Programa de Posgrado en Ciencias Marinas. A la Dra. MA Liceaga-Correa y Dr. FAAbreu-Grobois por su dirección para la investigación de grado del autor, además de los doctores D Valdés-Lozano, PLArdisson-Herrera y JI EuánÁvila por proponer el tema y realizar la Evaluación de Conocimientos que el autor acreditó con esta investigación individual. También a los revisores y editora de la revista RBMO por sus sugerencias y correcciones al manuscrito.

\section{LITERATURA CITADA}

Álvarez-Acosta A. 2016. Influencia de la temperatura superficial del mar sobre la fenología reproductiva de la tortuga carey (Eretmochelys imbricata) en playas índice de la península de Yucatán, México. Tesis de Maestría, Centro de Investigación y de Estudios Avanzados del Instituto Politécnico Nacional Unidad Mérida, Mérida, 94 pp.

Avens L \& KJ Lohman. 2004. Navigation and seasonal migratory orientation in juvenile sea turtles. The Journal of Experimental Biology 207: 1771-1778.

Avise JC. 1998. Conservation genetics in the marine realm. The American Genetic Association 89: 377-382.

Barceló-Celis A. 2015. Evaluación de la paternidad múltiple mediante el uso de microsatélites en la tortuga blanca (Chelonia mydas) en El Cuyo, Yucatán, México. Tesis de Maestría, Centro de Investigación y de Estudios avanzados del Instituto Politécnico Nacional, Mérida, 73 pp.

Bass AL. 1996. Application of isolation by distance models to hawksbill turtle (Eretmochelys imbricata) nesting sites in the Caribbean. In: Bowen BW \& WN Witzell (eds). Proceedings of the International Symposium on Sea Turtle Conservation Genetics. NOAA Technical Memorandum, NMFS-SEFSC-396: 41-46.

Beyer HL, DT Haydon, JM Morales, JL Frair, M Hebblewhite, M Mitchell \& J Matthiopoulos. 2010. The interpretation of habitat preference metrics under useavailability designs. Philosophical Transactions of the Royal Society B 365: 2245-2254.

Bjorndal KA, M Chaloupka, VS Saba, CE Diez, RP van Dam, BH Krueger, JA Horrocks, AJB Santos, C Bellini, MAG Marcovaldi, M Nava, S Willis, BJ Godley, S Gore, LA Hawkes, A McGowan, MJ Witt, TB Stringell, A Sanghera, PB Richardson, AC Broderick, Q Phillips, MC Calosso, JAB Claydon, J Blumenthal, F Moncada, G Nodarse, Y Medina, SG Dunbar, LD Wood, CJ Lagueux, CL Campbell, AB Meylan, PA
Meylan, VR Burns-Perez, RA Coleman, S Strindberg, V Guzmán-H, KM Hart, MS Cherkiss, Z Hillis-Starr, IF Lundgren, RH Boulon, S Connett Jr, ME Outerbridge \& AB Bolten. 2016. Somatic growth dynamics of West Atlantic hawksbill sea turtles: a spatiotemporal perspective. Ecosphere 7(5): e01279. <doi: 10.1002/ ecs2.1279>

Blumenthal JM, FA Abreu-Grobois, TJ Austin, AC Broderick, MW Bruford, MS Coyne, G Abanks-Petrie, A Formia, PA Maylan, AB Meylan \& BJ Godley. 2009. Turtle groups or turtle soup: dispersal patterns of hawksbill turtles in the Caribbean. Molecular Ecology 18(23): 48414853.

Bowen BW \& SA Karl. 1997. Population genetics, phylogeography and molecular evolution. In: Lutz PL \& JA Musick (eds). The biology of sea turtles 1: 29-50. CRC Press, Boca Raton.

Bowen BW, WS Grant, Z Hillis-Starr, DJ Shaver, KA Bjorndal, AB Bolten \& AL Bass. 2007. Mixed-stock analysis reveals the migrations of juvenile hawksbill turtles (Eretmochelys imbricata) in the Caribbean Sea. Molecular Ecology 16: 49-60.

Boyce MS \& LL McDonald. 1999. Relating populations to habitats using resource selection functions. TRENDS in Ecology \& Evolution 14(7): 268-272.

Brill RW, GH Balazs, KN Holland, RKC Chang, S Sullivan \& JC George. 1995. Daily movements, habitat use and submergence intervals of normal and tumor-bearing juvenile green turtles (Chelonia mydas L.) within a foraging area in the Hawaiian Islands. Journal of Experimental Marine Biology and Ecology 185: 203-218.

Brothers JR \& KJ Lohman. 2015. Evidence for geomagnetic imprinting and magnetic navigation in the natal homing of sea turtles. Current Biology 25: 392-396.

Burskirk SW \& JJ Millspaugh. 2006. Metrics for studies of resource selection. The Journal of Wildlife Management 70(2): 358-365.

Byles RA. 1991. Telemetría por satélite de tortugas marinas. En: Frazier J, R Vázquez, E Galicia, R Durán \& L Capurro (eds). Memorias del IV Taller Regional sobre Programas de Conservación de Tortugas Marinas en la Península de Yucatán, Yucatán, México, pp. 27-36.

Cassini MH. 2013. Distribution ecology. From individual habitat use to species biogeographical range, $219 \mathrm{pp}$. Springer, New York.

Ceriani SA, JD Roth, CR Sasso, CM McClellan, MC James, HL Haas, RJ Smolowitz, DR Evans, DS Addison, DA Bagley, LM Ehrhart \& JF Weishampel. 2014. Modeling and mapping isotopic patterns in the Northwest Atlantic derived from loggerhead sea turtles. Ecosphere 5(9): 122 <doi:10.1890/ES14-00230.1>

Cuevas E, MA Liceaga-Correa \& M Garduño-Andrade. 2007. Spatial characterization of a foraging area for immature hawksbill turtles (Eretmochelys imbricata) in Yucatan, Mexico. Amphibia-Reptilia 28: 337-346. 
Cuevas E, FA Abreu-Grobois, V Guzmán-Hernández, MA Liceaga-Correa \& RP Van Dam. 2008. Post-nesting migratory movements of hawksbill turtles Eretmochelys imbricata in waters adjacent to the Yucatan Peninsula, Mexico. Endangered Species Research 10: 123-133.

Cuevas E, MA Liceaga-Correa \& I Mariño-Tapia. 2010. Influence of beach slope and width on Hawksbill (Eretmochelys imbricata) and Green Turtle (Chelonia mydas) nesting activity in El Cuyo, Yucatán, Mexico. Chelonian Conservation and Biology 9(2): 262-267.

Davis JM \& JA Stamps. 2004. The effect of natal experience on habitat preferences. TRENDS in Ecology and Evolution 19(8): 411-416.

Diego-Rasilla FJ. 2004. El sentido magnético y su uso en la orientación de los animales. In: Pereira D, MA Bárcena, I Rubio \& J Sesma (eds). Aproximación a las ciencias planetarias, pp. 269-297. Ediciones Universidad de Salamanca, Salamanca.

Engstrom TN, PA Meylan \& AB Meylan. 2002. Origin of juvenile loggerhead turtles (Caretta caretta) in a tropical development habitat in Caribbean Panamá. Animal Conservation 5: 125-133.

Feldheim KA, SH Gruber, JD Dibattista, EA Babcock, ST Kessel, AP Hendry, EK Pikitch, MV Ashley \& DD Chapman. 2014. Two decades of genetic profiling yields first evidence of natal philopatry and long-term fidelity to parturition sites in sharks. Molecular Ecology 23(1): 110-117. <doi: 10.1111/mec.12583>

Ficetola GF. 2007. The influence of beach features on nesting of the hawksbill turtle Eretmochelys imbricata in the Arabian Gulf. Oryx 41(3): 402-405.

Gilroy JJ \& WJ Sutherland. 2007. Beyond ecological traps: perceptual errors and undervalued resources. TRENDS in Ecology and Evolution 22(7): 351-356.

Goatley CHR, AS Hoey \& DR Bellwood. 2012. The role of turtles as coral reef macroherbivores. PLoS ONE 7(6): e39979 <doi: 10.1371/journal.pone.0039979>

Godley BJ, JM Bluementhal, AC Broderick, MS Coyne, MH Godfrey, LA Hawkes \& MJ Witt. 2007. Satellite tracking of sea turtles: where have we been and where do we go next? Endangered Species Research 3: 1-20.

González-Garza BI, A Stow, LF Sánchez-Teyer \& O ZapataPérez. 2015. Genetic variation, multiple paternity, and measures of reproductive success in the critically endangered hawksbill turtle (Eretmochelys imbricate). Ecology and Evolution 5(24): 5758-5769.

Goodchild, MF \& DA Quattrochi. 1997. Scale, multiscaling, remote sensing and GIS. In: Quattrochi DA \& MF Goodchild (eds). Scale in remote sensing and GIS, pp. 1-12. Lewis Publishers, Washington D.C.

Gulko DA \& KL Eckert. 2004. Sea turtles: An ecological guide, 122 pp. WIDECAST Mutual Publishing, Honolulu.
Hawkes LA, AC Broderick, MS Coyne, MH Godfrey, LF Lopez-Jurado, P Lopez-Suarez, SE Merino, N VaroCruz \& BJ Godley. 2006. Phenotypically linked dichotomy in sea turtle foraging requires multiple conservation approaches. Current Biology 16: 990-995.

Hawkes LA, AC Broderick, MH Godfrey \& BJ Godley. 2007. Investigating the potential impacts of climate change on a marine turtle population. Global Change Biology 13: 110.

Heithaus MR, IM Hamilton, AJ Wirsing \& LM Dill. 2006. Validation of a randomization procedure to assess animal habitat preferences: microhabitat use of tiger sharks in a seagrass ecosystem. Journal of Animal Ecology 75: 666-676.

Horrocks B \& NM Scott. 1991. Nest site location and nest success in the Hawksbill turtle in Barbados. Marine Ecology Progress Series 69: 1-8.

IUCN. 2017. The IUCN Red List of Threatened Species. International Union for Conservation of Nature and Natural Resources. <http://www.iucnredlist.org/>

Jeffers VF \& BJ Godley. 2016. Satellite tracking in sea turtles: How do we find our way to the conservation dividends? Biological Conservation 199: 172-184.

Julliard R. 2000. Sex-specific dispersal in spatially varying environments leads to habitat-dependent evolutionary stable offspring sex ratios. Behavioral Ecology 11(4): 421-428.

Kamel SJ \& N Mrosovsky. 2006. Inter-seasonal maintenance of individual nest site preferences in hawksbill sea turtles. Ecology 87(11): 2947-2952.

Kelly I, JX Leon, BL Gilby, AD Olds \& TA Schlacher. 2017. Marine turtles are not fussy nesters: a novel test of smallscale nest site selection using structure from motion beach terrain information. PeerJ 5: e2770. <doi:10.7717/peerj.2770>

Lahanas PN, KA Bjorndal, AB Bolten, SE Encalada, MM Miyamoto, RA Valverde \& BW Bowen. 1998. Genetic composition of a green turtle (Chelonia mydas) feeding ground population: evidence for multiple origins. Marine Biology 130: 345-352.

Lee PLM, P Luschi \& GC Hays. 2007. Detecting female precise natal philopatry in green turtles using assignment methods. Molecular Ecology 16: 61-74.

León YM \& KA Bjorndal. 2002. Selective feeding in the hawksbill turtle, an important predator in coral reef systems. Marine Ecology and Progress Series 245: 249-258.

Lohman KJ, CMF Lohman, JR Brothers \& NF Putman. 2003. Natal homing and imprinting in sea turtles. In: Wyneken J, KJ Lohman \& JA Musick (eds). The biology of sea turtles, Volume 3: 59-78. CRC Press, Boca Raton.

Lohman KJ, CMF Lohman \& NF Putman. 2007. Magnetic maps in animals: nature's GPS. The Journal of Experimental Biology 210: 3697-3705.

Lohman KJ, NF Putman \& CMF Lohman. 2012. The 
magnetic map of hatchling loggerhead sea turtles. Current Opinion in Neurobiology 22: 336-342.

Luschi P, S Benhamou, C Girard, S Ciccione, D Roos, J Sudre \& S Benvenuti. 2007. Marine turtles use geomagnetic cues during open-sea homing. Current Biology 17(2): 126-133.

Manly BFJ, LL McDonal, DL Thomas, TL McDonald \& WP Erickson. 2002. Resource selection by animals, 221 pp. Kluwer Academic Publishers, Amsterdam.

Mazaris AD, YG Matsinos \& D Margaritoulis. 2006. Nest site selection of loggerhead sea turtles: the case of the island of Zakynthos, W. Greece. Journal of Experimental Marine Biology and Ecology 336: 157-162.

Mazaris AD, AS Kallimanis, JD Pantis \& GC Hays. 2013. Phenological response of sea turtles to environmental variation across a species' northern range. Proceedings of the Royal Society of London B: Biological Sciences 280(1751): <doi:10.1098/rspb.2012.2397>

Méndez D, E Cuevas, J Navarro, BI González-Garza \& V Guzmán-Hernández. 2013. Rastreo satelital de tortugas blanca hembra Chelonia mydas, y evaluación de sus ámbitos hogareños en la costa norte de la península de Yucatán, México. Revista de Biología Marina y Oceanografía 48(3): 497-509.

Mortimer JA. 1990. The influence of beach sand characteristics on the nesting behavior and clutch survival of green turtles (Chelonia mydas). Copeia 3: 802-817.

Neeman N, NJ Robinson, FV Paladino, JR Spotila \& MP O'Connor. 2015. Phenology shifts in leatherback turtles (Dermochelys coriacea) due to changes in sea surface temperature. Journal of Experimental Marine Biology and Ecology 462: 113-120.

Neuhauser C. 2001. Mathematical challenges in spatial ecology. Notices of the American Mathematical Society 48(11): 13041314.

Nishizawa H, O Abe, J Okuyama, M Kobayashi \& N Arai. 2011. Population genetic structure and implications for natal philopatry of nesting green turtles Chelonia mydas in the Yaeyama Islands, Japan. Endangered Species Research 14: 141-148

Norrgard JW \& JE Graves. 1996. Determination of the natal origin of juvenile loggerhead turtle (Caretta caretta) populations in Chesapeake Bay using mitochondrial DNA analysis. In: Bowen BW \& WN Witzell (eds). Proceedings of the International Symposium on Sea Turtle Conservation Genetics, NOAA Technical Memorandum, NMFS-SEFSC396: 129-138.

Pech D, PL Ardisson, E Bourget \& AR Condal. 2007. Abundance variability of benthic intertidal species: effects of changing scale on patterns perception. Ecography 30: 637648.
Pereira GM. 2002. A typology of spatial and temporal scale relations. Geographical Analysis 34(1): 21-33.

Pfaller JB, CJ Limpus \& KA Bjorndal. 2008. Nest-site selection in individual loggerhead turtles and consequences for doomed-egg relocation. Conservation Biology 23(1): 7280.

Provancha JA \& LM Ehrhart. 1987. Sea turtle nesting trends at Kennedy Space Center and Cape Canaveral air force station, Florida, and relationships with factors influencing nest site selection. In: Witzel WN (ed). Ecology of east Florida sea turtles. U. S. Department of Commerce, NOAA Technical Report NMFS 53: 33-44.

Putman NF, P Verley, TJ Shay \& KJ Lohman. 2012. Simulating transoceanic migrations of young loggerhead sea turtles: merging magnetic navigation behavior with an ocean circulation model. The Journal of Experimental Biology 215: 1863-1870.

Putman NF, FA Abreu-Grobois, AC Broderick, C Ciofi, A Formia, BJ Godley, S Stroud, T Pelembe, P Verley \& N Williams. 2014. Numerical dispersal simulations and genetics help explain the origin of hawksbill sea turtles in Ascension Island. Journal of Experimental Marine Biology and Ecology 450: 98-108.

Rees AF, J Alfaro-Shigueto, PCR Barata, KA Bjorndal, AB Bolten, J Bourjea, AC Broderick, LM Campbell, L Cardona, C Carreras, $\mathbf{P}$ Casale, SA Ceriani, PH Dutton, T Eguchi, A Formia, MMPB Fuentes, WJ Fuller, M Girondot, MGH Godfrey, M Hamann, KM Hart, GC Hays, S Hochscheid, Y Kaska, MP Jensen, JC Mangel, JA Mortimer, E Naro-Maciel, CKY Ng, WJ Nichols, AD Phillot, RD Reina, O Revuelta, G Schofield, JA Seminoff, K Shanker, J Tomás, JP Van der Merwe, KS Van Houtan, HN Van der Zanden, BP Wallace, KR Wedemeyer-Strombel, TM Work \& BJ Godley. 2016. Are we working towards global research priorities for management and conservation of sea turtles? Endangered Species Research 31: 337-382.

Refsnider JM \& FJ Janzen. 2010. Putting eggs in one basket: Ecological and evolutionary hypotheses for variation in oviposition-site choice. Annual Review of Ecology, Evolution and Systematics 41: 39-57.

Renaud ML \& JA Carpenter. 1994. Movements and submergence patterns of Loggerhead turtles (Caretta caretta) in the Gulf of Mexico determined through satellite telemetry. Bulletin of Marine Science 55(1): 1-15.

Rietkerk M, J van de Koppel, L Kumar \& F van Langevelde. 2002. The ecology of scale. Ecological Modelling 149: 1-4.

Rumbold DG, PW Davis \& C Perretta. 2001. Estimating the effect of beach nourishment on Caretta caretta (Loggerhead sea turtle) nesting. Restoration Ecology 9(3): 304-310. 
Schlaepfer MA, MC Runge \& PW Sherman. 2002. Ecological and evolutionary traps. TRENDS in Ecology \& Evolution 17(10): 474-480.

Seminoff JA, A Resendiz \& WJ Nichols. 2002. Home range of green turtles Chelonia mydas at a coastal foraging area in the Gulf of California, Mexico. Marine Ecology Progress Series 242: 253-265.

Shaver DJ, KM Hart, I Fujisaki, C Rubio, AR SartainIverson, J Peña, D Gomez-Gamez, RJ González-DíazMirón, PM Burchfield, HJ Martínez \& J Ortíz. 2016. Migratory corridors of adult female Kemp's ridley turtles in the Gulf of Mexico. Biological Conservation 194: 158-167.

Stiebens VA, SE Merino, C Roder, FJJ Chain, PLM Lee \& C Eizaguirre. 2013. Living on the edge: how philopatry maintains adaptive potential. Proceedings of the Royal Society B 280: <doi:10.1098/rspb.2013.0305>

Switzer PV. 1993. Site fidelity in predictable and unpredictable habitats. Evolutionary Ecology 7: 533-555.

Tiwari M, KA Bjorndal, AB Bolten \& BM Bolker. 2005. Intraspecific application of the mid-domain effect model:spatial and temporal nest distributions of green turtles, Chelonia mydas, at Tortuguero, Costa Rica. Ecology Letters 8: 918-924.
Turner SJ, RV O’Neill, W Conley, MR Conley \& HC Humpries. 1999. Pattern and scale: statistics for landscape ecology. In: Turner MG \& RH Gardner (eds). Quantitative methods in landscape ecology, pp. 17-50. Springer-Verlag, Nueva York.

Valenzuela N \& FJ Janzen. 2001. Nest-site philopatry and the evolution of temperature-dependent sex determination. Evolutionary Ecology Research 3: 779-794.

Van Dam RP \& CE Diez. 1996. Diving behavior of immature hawksbills (Eretmochelys imbricata) in a Caribbean Cliff wall habitat. Marine Biology 127(1): 171-178.

Vander-Zanden HB, AD Tucker, AB Bolten, KJ Reich \& KA Bjorndal. 2014. Stable isotopic comparison between loggerhead sea turtle issues. Rapid Communications in Mass Spectrometry 28: 2059-2064.

Wood DW \& KA Bjorndal. 2000. Relation of temperature, moisture, salinity and slope to nest site selection in Loggerhead sea turtles. Copeia 2000(1): 119-128.

Recibido el 27 de marzo de 2017 y aceptado el 15 de junio de 2017

Editor: Claudia Bustos D. 